\title{
Validation of the Enhanced Recovery after Surgery (ERAS) society recommendations for liver surgery: a prospective, observational study
}

\author{
Moritz Schmelzle ${ }^{1 \#}$, Felix Krenzien ${ }^{1,2 \#}$, Paul Dahlke ${ }^{1}$, Alina Krombholz ${ }^{1}$, Nora Nevermann ${ }^{1}$, \\ Linda Feldbrügge ${ }^{1}$, Axel Winter ${ }^{1}$, Wenzel Schöning ${ }^{1}$, Christian Benzing ${ }^{1}$, Johann Pratschke ${ }^{1}$, \\ Jens Neudecker ${ }^{1}$ \\ ${ }^{1}$ Department of Surgery, Campus Charité Mitte and Campus Virchow-Klinikum, Charité-Universitätsmedizin, corporate member of Freie \\ Universität Berlin, Humboldt-Universität zu Berlin, Berlin, Germany; ${ }^{2}$ Berlin Institute of Health (BIH), Berlin, Germany \\ Contributions: (I) Conception and design: F Krenzien, J Neudecker, M Schmelzle; (II) Administrative support: J Neudecker, J Pratschke; (III) \\ Provision of study materials or patients: A Winter, A Krombholz, L Feldbrügge; (IV) Collection and assembly of data: P Dahlke, J Neudecker; (V) \\ Data analysis and interpretation: F Krenzien, P Dahlke; (VI) Manuscript writing: All authors; (VII) Final approval of manuscript: All authors. \\ \#These authors contributed equally to this work. \\ Correspondence to: Prof. Dr. med. Moritz Schmelzle. Department of Surgery, Campus Charité Mitte and Campus Virchow-Klinikum, Charité \\ Universitätsmedizin Berlin, Augustenburger Platz 1, 13353 Berlin, Germany. Email: moritz.schmelzle@charite.de.
}

Background: Twenty-three recommendations were summarized by the Enhanced Recovery After Surgery (ERAS) society for liver surgery. The aim was to validate the protocol especially with regard to adherence and the impact on morbidity.

Methods: Using the ERAS Interactive Audit System (EIAS), ERAS items were evaluated in patients undergoing liver resection. Over a period of 26 months, 304 patients were prospectively enrolled in an observational study (DRKS00017229). Of those, 51 patients (non-ERAS) were enrolled before and 253 patients (ERAS) after the implementation of the ERAS protocol. Perioperative adherence and complications were compared between the two groups.

Results: Overall adherence increased from $45.2 \%$ in the non-ERAS group to $62.7 \%$ in the ERAS group $(\mathrm{P}<0.001)$. This was associated with significant improvements in the preoperative and postoperative phase $(\mathrm{P}<0.001)$, rather than in the outpatient and intraoperative phase (both $\mathrm{P}>0.05)$. Overall complications decreased from $41.2 \%(n=21)$ in the non-ERAS group to $26.5 \%(n=67)$ in the ERAS group $(P=0.0423)$, which was mainly due to the reduction of grade $1-2$ complications from $17.6 \%(n=9)$ to $7.6 \%(n=19)$ $(\mathrm{P}=0.0322)$. As for patients undergoing open surgery, implementation of ERAS lead to a reduction of overall complications in patients scheduled for minimally invasive liver surgery (MILS) ( $\mathrm{P}=0.036)$.

Conclusions: Implementation of the ERAS protocol for liver surgery according to the ERAS guidelines of the ERAS Society reduced Clavien-Dindo grade 1-2 complications particularly in patients who underwent MILS. The ERAS guidelines are beneficial for the outcome, while adherence to the various items has not yet been satisfactorily defined.

Keywords: Enhanced Recovery After Surgery (ERAS); prehabilitation; liver surgery

Submitted Jul 16, 2021. Accepted for publication Nov 16, 2021. Published online Feb 09, 2022.

doi: $10.21037 / \mathrm{hbsn}-21-294$

View this article at: https://dx.doi.org/10.21037/hbsn-21-294 


\section{Introduction}

The Enhanced Recovery After Surgery (ERAS) society, founded in 2010 (http://www.erassociety.org), developed and published several evidence-based consensus protocols for patients undergoing surgery (1). ERAS programs have been introduced to reduce the perioperative stress response and to improve perioperative outcomes by reducing postoperative complications, accelerating functional recovery and decreasing duration of postoperative stay.

The ERAS society published the first ERAS guidelines on liver surgery in 2016 (2). The guidelines have been developed by a two-step process. First, a systematic review was made validating the classical 23 ERAS items for evidence in liver surgery. Second, 7 selected experts reviewed the findings in a modified Delphi process and rated the items according to level of evidence and grade of recommendation. The goal was to find the best available evidence for its use as an item in daily clinical routine. At this time, only 16 of 23 items were supported by any evidence from studies in liver surgery, while the remaining 7 items were mostly tested in colorectal surgery (2). This process formed the ERAS guidelines for liver surgery which have been used since then by several groups (3-7).

The guidelines are supposed to improve the perioperative management in liver surgery, while its strict application and the adherence of the patients remains vague in many clinical trials (8). Consequently, reports on ERAS implementation in liver surgery are mixed with regards to length of stay, postoperative complications, and hospital costs (8). Protocols are often adapted due to the interaction between the department of anaesthesiology and surgery. The ERAS Society demanded the continuous auditing of the patients to assess their adherence to the protocol (adherence), when they published the guideline (2). However, this has not been addressed in any study yet. Furthermore, there has been a paradigm shift in liver surgery in recent years from open to minimal invasive approaches (9-11). The concept of a minimally invasive liver surgery (MILS) has become standardized, resulting in a much lower surgical trauma and less complications (9). In this respect, the next question arise whether these patients might benefit from the implementation of ERAS items to the same extent as postulated for patients after open liver resection.

The aim of this prospective study was to assess the adherence and surgical outcome of patients undergoing liver resection after implementation of the 23 items of the ERAS guidelines for open liver surgery and MILS.
We present the following article in accordance with the STROBE reporting checklist (available at https:// hbsn.amegroups.com/article/view/10.21037/hbsn-21294/rc) (12).

\section{Methods}

This prospective observational cohort study was approved by the local ethics committee of the CharitéUniversitätsmedizin Berlin under the registration numbers EA2/108/18 and EA4/153/18, and was registered with the German Clinical Trials Register (No. DRKS00011631). All study participants gave written informed consent for study inclusion. The study was conducted in accordance with the Declaration of Helsinki (as revised in 2013). The ERAS program was implemented at the Department of Surgery, Campus Charité Mitte and Campus Virchow-Klinikum, Charité-Universitätsmedizin Berlin. From July 2018 to March 2020, 304 patients were consecutively included. Of these, 51 patients were treated according to the clinic standard at that time from July 2018 to February 2019 and were included in this study as a control group (non-ERAS cohort). After the ERAS implementation process, from March 2019 to September 2020, 253 patients were treated according to the ERAS protocol and included in the study as a treatment group (ERAS cohort). No patients have been excluded from analysis.

\section{ERAS program}

An ERAS core team with surgeons, anesthesiologists, physiotherapists was formed for the implementation process. An extra ERAS study nurse was hired for the program and ERAS patient care. The ERAS core team then drafted ERAS-specific standard operating procedures in addition to patient information brochures and patient diaries to better implement in each perioperative phase. The ERAS program began in March 2019 with several specific interdisciplinary ERAS training sessions and distribution of informational materials (patient diary, patient information brochure). Data for the present analysis were prospectively entered into the ERAS Interactive Audit System (EIAS) (Encare, Stockholm, Sweden); pre-, intra-, and postoperative data and complications were documented during the hospital stay. Patients who had already been discharged were contacted by telephone 30 days after surgery. 


\section{Inclusion criteria}

Patients who were at least 18 years old and planned for elective liver resection were included in this study. In addition, written informed consent had to be obtained from the patient before study inclusion.

\section{Exclusion criteria}

Patients who underwent emergency surgery and patients who did not provide written informed consent for participation in the study and for the storage of personal data were excluded from the study.

\section{ERAS protocol}

The applied ERAS protocol was based on the official ERAS guidelines of the ERAS society (2). The individual ERAS items applied and their corresponding guideline counterparts are listed in Table 1. Patients were considered adherent to the respective ERAS item, if this item was applicable to them and the corresponding adherence conditions of the EIAS database were met. The patient data included age, gender, height, weight and nutritional status according to Nutritional Risk Screening (13). In addition, risk factors such as known type II diabetes (DMII), alcohol consumption and smoking behavior, cardiac and respiratory diseases requiring treatment and neoadjuvant therapies that could reduce liver function were documented. Individual liver function parameters such as the serum bilirubin concentration (biotransformation function), and the thromboplastin time/international normalized ratio (INR; coagulation) were also documented. For fluid balancing, the amount and duration of postoperative intravenous fluid substitution were documented. Morning weight, oral fluid intake, and caloric intake from sip feeds were also recorded on postoperative day (POD) 1, POD2 and POD3. In case of an abdominal drain, output was documented day by day. The duration of mobilization was documented daily. If the indwelling urinary catheter (IUC) was not removed at the end of the surgery, it was also documented when it was removed postoperatively.

\section{Adberence}

The adherence rates of the patients were calculated by forming the rate of the adherence items fulfilled (Table 1). If an item was not applicable, the item was not included in the calculation of the adherence rate. In these cases, the number of adherence items to be fulfilled was reduced accordingly. In this way, both the adherence rates in the individual perioperative phases and the overall adherence rate were calculated.

\section{MILS}

The standard procedure of liver resection was minimally invasive. If any of the following characteristics were present, open procedure was performed:

* Excessive adhesions due to previous surgery;

* Bilidigestive reconstructions;

* Vascular reconstructions.

\section{Propensity score matching (PSM)}

PSM and statistical analysis were performed using $\mathrm{R}$ (version 4.0.2; R Foundation for Statistical Computing, Vienna, Austria). The score was calculated using a logit model (package "MatchIt") based on the following parameter: age, sex, body mass index (BMI) $\left(\mathrm{kg} / \mathrm{m}^{2}\right)$, preoperative chemotherapy, preoperative radiotherapy, history of surgery (right upper abdomen), serum bilirubin (mmol/L), American Society of Anesthesiologists classification (ASA 3-4 =1; ASA 1-2 =0), extent of resection (major resection $=1$; minor resection $=0$ ), and dignity of resection (malignant $=1$; benign $=0$ ). "Nearest Neighbor" was used as the distance metric for all match procedures, and pairing was performed without replacement for all match procedures. A 1:n match was applied. Absolute standardized mean differences (SMD) were used for balance diagnostics of covariates. All SMD used in this paper are understood to be absolute values. The SMD should be $<0.2$ per parameter, if possible, to argue for balanced covariate expression between cohorts.

\section{Statistics}

The comparison of differences of baseline characteristics between the total cohorts was performed using SMD. An SMD of 0.2 was chosen as the limit of effect size according to Cohen. Thus, an SMD $\geq 0.2$ was considered to indicate an unbalanced distribution between the two cohorts, whereas an $\mathrm{SMD}<0.2$ was considered to indicate a balanced distribution between the two cohorts. Missing values were not imputed. For the analyses of matched cohorts, generalized estimating equations (GEEs) were used to test for differences in complication rates as well as adherence. 
Table 1 ERAS protocol and adherence conditions

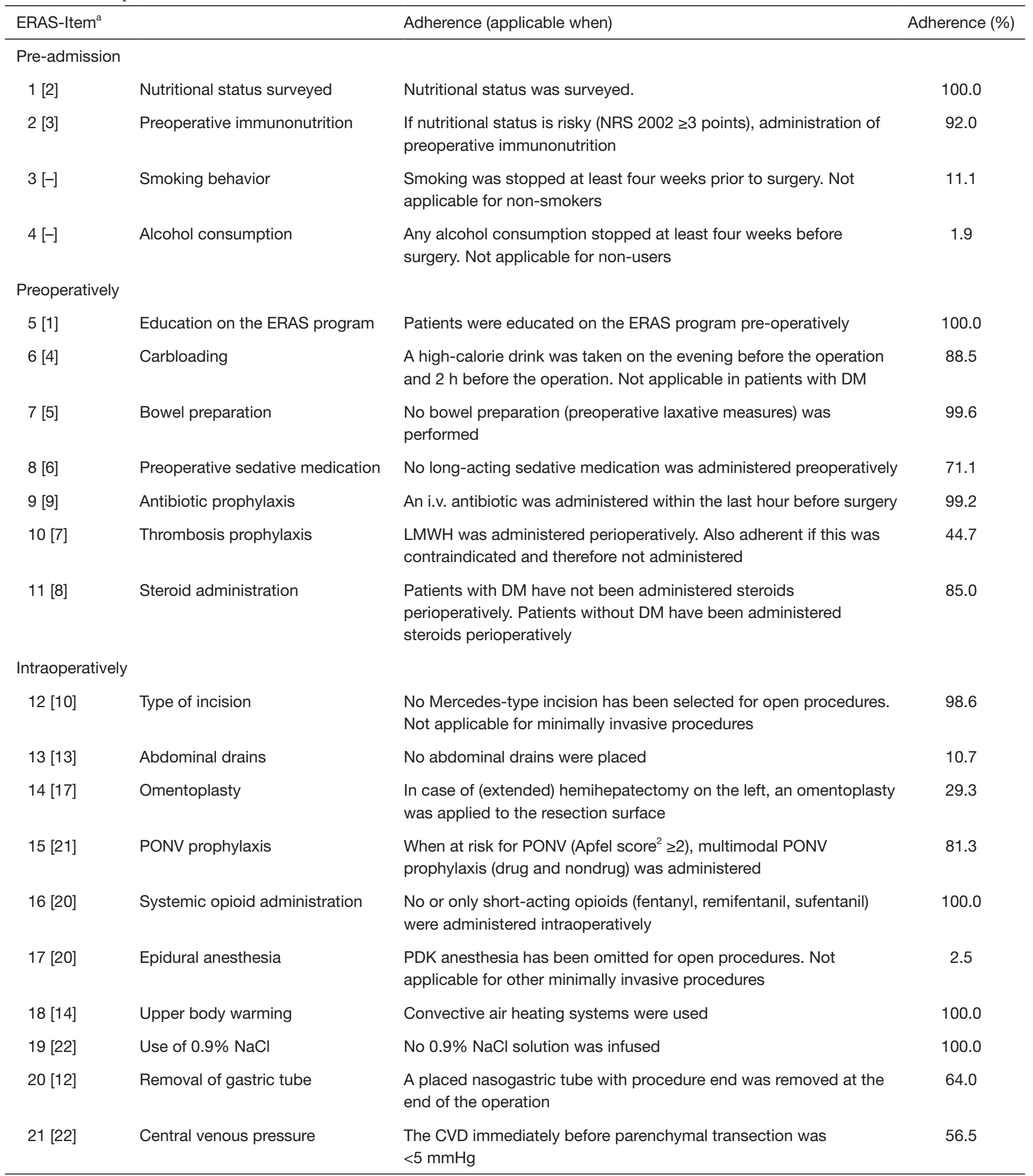

Table 1 (continued) 
Table 1 (continued)

\begin{tabular}{|c|c|c|c|}
\hline ERAS-Item ${ }^{a}$ & & Adherence (applicable when) & Adherence (\%) \\
\hline \multicolumn{4}{|c|}{ Postoperatively } \\
\hline 22 [22] & $\begin{array}{l}\text { Termination of i.v. fluid } \\
\text { administration }\end{array}$ & i.v. fluid delivery was stopped after $\leq 1$ night & 60.1 \\
\hline 23 [22] & Postoperative weight gain & $\begin{array}{l}\text { The weight gain from pre- to postoperative was a maximum of } 2 \mathrm{~kg} \text {. } \\
\text { Thus, fluid balance was verified }\end{array}$ & 22.9 \\
\hline $25[15]$ & Energy consumption at POD1 & On day 1 postoperatively, $\geq 600 \mathrm{kcal}$ were consumed via sip feeds & 14.2 \\
\hline 26 [19] & Mobilization on the day of surgery & $\begin{array}{l}\text { On the day of surgery postoperatively, any mobilization has taken } \\
\text { place outside the bed }\end{array}$ & 23.3 \\
\hline $30[-]$ & Removal of indwelling catheter & IUC was removed after one night at the latest & 77.8 \\
\hline $31[16]$ & Control postoperative glycemia & Postoperative control of blood glucose has taken place & 84.2 \\
\hline 32 [20] & Postoperative PDK & $\begin{array}{l}\text { PDK anesthesia was also dispensed with postoperatively for open } \\
\text { procedures. Not applicable for other minimally invasive procedures }\end{array}$ & 67.7 \\
\hline $33[-]$ & 30 days follow-up & $\begin{array}{l}\text { Patients were contacted } 30 \text { days after surgery to document any } \\
\text { post-inpatient complications }\end{array}$ & 81.3 \\
\hline
\end{tabular}

Patients were considered adherent to the respective ERAS item if this ERAS item was applicable to them and the corresponding adherence conditions of the EIAS database were met. a , first number represents the item applied in this ERAS protocol (33 items), number in square brackets represents the corresponding guideline items (23 items; empty if no corresponding guideline item exists). ERAS, Enhanced Recovery After Surgery; NRS 2002, Nutritional Risk Screening (13); DM, diabetes mellitus; i.v., intravenous; LMWH, low-molecular-weight heparin; PONV, postoperative nausea and vomiting; PDK, peridural catheter; NaCl, sodium chloride; CVD, central venous pressure; $\mathrm{POD}(\mathrm{X})$, postoperative day X; IUC, indwelling urinary catheter; EIAS, ERAS Interactive Audit System.

GEEs were furthermore used to calculate odds ratios (OR) and $95 \%$ confidence intervals ( $95 \% \mathrm{CI}$ ) for complication rates and 95\% CI for differences in adherence at each perioperative stage and overall. For analyses of unmatched cohorts, Fisher's exact test was used to test for differences in complication rates whereas Welch's test was used to test for differences in adherence. To display the results of the adherence, a heat map was compiled by Ward minimum variance hierarchical clustering with Euclidean distance. The significance level ( $\alpha$-level) chosen was 0.05 .

\section{Results}

\section{Patients characteristics}

Over a period of 26 months, 304 patients were prospectively included in the analysis before liver resection, of which
51 patients $(17 \%)$ served as a control group before the implementation of the ERAS protocol (non-ERAS), whereas the ERAS protocol was subsequently applied in 253 consecutive patients (83\%, ERAS). In all patients, perioperative adherence of each ERAS item and postoperative complications were recorded and compared between the two groups before and after implementation of ERAS.

\section{Clinical characteristics}

Sex, BMI, DMII, excessive alcohol intake, smoking and performance status (WHO and ASA), were comparable in the non-ERAS group to the ERAS group (Table 2). Also, there were no differences between the groups in the rate of pretreated patients (radio- or chemotherapy). However, 
Table 2 Baseline characteristics of the study cohort

\begin{tabular}{|c|c|c|c|c|}
\hline Parameter & Non-ERAS $(\mathrm{N}=51)$ & ERAS $(\mathrm{N}=253)$ & SMD & $\mathrm{P}$ \\
\hline Age (years) & $62.75 \pm 15.37$ & $61.40 \pm 13.23$ & 0.094 & 0.561 \\
\hline $\mathrm{BMI}\left(\mathrm{kg} / \mathrm{m}^{2}\right)$ & $26 \pm 4.70$ & $2,611 \pm 4.87$ & 0.022 & 0.884 \\
\hline$<18.5$ & $2(3.9)$ & $5(2.0)$ & 0.262 & 0.325 \\
\hline $25-<30$ & $19(37.3)$ & $114(45.1)$ & & \\
\hline$\geq 30$ & $21(41.2)$ & $78(30.8)$ & & \\
\hline Neoadjuvant chemotherapy & $16(31.4)$ & $90(35.6)$ & 0.089 & 0.631 \\
\hline Neoadjuvant radiotherapy & $7(13.7)$ & $10(4.0)$ & 0.35 & 0.851 \\
\hline Daily $>3$ standard glasses of alcohol before surgery & $6(11.8)$ & $32(12.6)$ & 0.027 & 1.000 \\
\hline \multicolumn{5}{|l|}{ Diabetes mellitus } \\
\hline No diabetes mellitus & $41(80.4)$ & $216(85.4)$ & 0.272 & 0.378 \\
\hline No drugs & $0(0.0)$ & $5(2.0)$ & & \\
\hline On drugs & $10(19.6)$ & $32(12.6)$ & & \\
\hline \multicolumn{5}{|l|}{ WHO-performance status } \\
\hline Asymptomatic & $48(94.1)$ & $222(87.7)$ & 0.247 & 0.609 \\
\hline Symptomatic, mobile & $3(5.9)$ & $28(11.1)$ & & \\
\hline ASA 1-2 & $27(52.9)$ & $112(44.3)$ & 0.174 & 0.283 \\
\hline ASA 3-4 & $24(47.1)$ & $141(55.7)$ & & \\
\hline \multicolumn{5}{|l|}{ Liver disease } \\
\hline Benign liver lesion & $8(15.7)$ & $33(13.0)$ & 0.334 & 0.082 \\
\hline Primary liver carcinoma & $19(37.3)$ & $93(36.8)$ & & \\
\hline Secondary liver carcinoma & $20(39.2)$ & $123(48.6)$ & & \\
\hline Other & $4(7.8)$ & $4(1.6)$ & & \\
\hline
\end{tabular}

Table 2 (continued) 
Table 2 (continued)

\begin{tabular}{|c|c|c|c|c|}
\hline Parameter & Non-ERAS $(\mathrm{N}=51)$ & ERAS $(\mathrm{N}=253)$ & SMD & $\mathrm{P}$ \\
\hline Hemihepatectomy left & $9(17.6)$ & $38(15.0)$ & 0.222 & 0.969 \\
\hline Extended hemihepatectomy left & $5(9.8)$ & $22(8.7)$ & & \\
\hline Hemihepatectomy right & $5(9.8)$ & $37(14.6)$ & & \\
\hline Sectionectomy, segmentectomy & $23(45.1)$ & $116(45.8)$ & & \\
\hline Wedge resection & $5(9.8)$ & $15(5.9)$ & & \\
\hline \multicolumn{5}{|l|}{ Extend of resection } \\
\hline Minor & $26(51.0)$ & 97 (38.3) & 0.258 & 0.284 \\
\hline \multicolumn{5}{|l|}{ Approach } \\
\hline Open surgery & $17(33.3)$ & $77(30.4)$ & 0.062 & 0.740 \\
\hline Minimal-invasive & $34(66.7)$ & $176(69.6)$ & & \\
\hline Length of stay (days) & $7[5.5-9]$ & 7 [5-9] & 0.207 & 0.643 \\
\hline
\end{tabular}

The data are presented as mean \pm SD, median [IQR], or $n(\%)$. Chi ${ }^{2}$-test or exact Fisher-test for categorical variables, Welch's $t$-test or Wilcoxon rank-sum test for continuous variables. ERAS, Enhanced Recovery After Surgery; SMD, standardized mean difference; BMI, body mass index; WHO, World Health Organization; INR, international normalized ratio; ASA, American Society of Anesthesiologists; IQR, interquartile range.

there was a higher rate of patients with a history of previous surgery (in the right upper abdomen) in the ERAS group (41.9\% ERAS vs. $21.6 \%$ pre-ERAS, $\mathrm{P}=0.007$ ), which may be associated with a trend towards more secondary liver tumors, e.g., colorectal liver metastases (48.6\% ERAS vs. $39.2 \%$ non-ERAS). The INR was significantly increased in the ERAS group, but the difference was not clinically relevant at 1.06 vs. $1.01(\mathrm{P}=0.003)$. Other laboratory chemical parameters were comparable between both groups.

\section{Surgical characteristics}

With regard to surgical technical aspects, there were no differences between the non-ERAS and the ERAS group. Both the type of surgery and the extent of resection were comparable. A total of 34 patients $(66.7 \%)$ in the control group and 176 patients $(69.6 \%)$ in the ERAS group underwent MILS.

\section{Overall adberence}

Adherence to items required in the ERAS protocol was
$45.2 \%$ at our center even before the ERAS program was implemented. After implementation of the ERAS program, overall adherence was increased to $62.7 \%$. This improvement of $17.5 \%$ was statistically significant $(\mathrm{P}<0.001$, Table 3). Perioperative adherence broke down as follows: $86.3 \%$ pre-admission, $59.2 \%$ preoperative, $71.8 \%$ intraoperative, $20.1 \%$ postoperative in the nonERAS group and $86.9 \%$ pre-admission $(\mathrm{P}=0.866), 83.8 \%$ preoperative $(\mathrm{P}<0.001), 71.6 \%$ intraoperative $(\mathrm{P}=0.913)$, $43.9 \%$ postoperative $(\mathrm{P}<0.001)$ in the ERAS group.

Next, we conducted a 1:n-PSM to estimate the effect of ERAS by accounting for covariates that predict receiving the treatment (Table 4). The SMD was $<0.2$ per parameter, indicating balanced expression of the covariates between cohorts (Figure 1). In total, 47 non-ERAS patients were matched with 200 ERAS patients. With ERAS implementation, the adherence increased preoperatively, postoperatively and overall highly significantly (Figure 2). In contrast, pre-admission adherence and intraoperative adherence did not change $(\mathrm{P}>0.05)$.

We next examined whether adherence differed within the ERAS cohort depending on patient characteristics and 
Table 3 Event rates and perioperative adherence of the overall cohort

\begin{tabular}{|c|c|c|c|c|}
\hline Parameter & Non-ERAS $(\mathrm{N}=51)$ & ERAS $(N=253)$ & SMD & $\mathrm{P}$ \\
\hline Clavien-Dindo 1-5 & $21(41.2)$ & $67(26.5)$ & 0.314 & 0.042 \\
\hline Clavien-Dindo 1-2 & $9(17.6)$ & $19(7.5)$ & 0.309 & 0.032 \\
\hline Clavien-Dindo 3-5 & $12(23.5)$ & $48(19.0)$ & 0.112 & 0.445 \\
\hline \multicolumn{5}{|l|}{ Adherence, \% } \\
\hline Pre-admission (mean $\pm \mathrm{SD}$ ) & $86.3 \pm 24.0$ & $86.9 \pm 22.9$ & 0.026 & 0.866 \\
\hline Preoperatively (mean \pm SD) & $59.2 \pm 10.3$ & $83.8 \pm 11.8$ & 2.215 & $<0.001$ \\
\hline Intraoperatively (mean \pm SD) & $71.8 \pm 12.1$ & $71.6 \pm 12.2$ & 0.017 & 0.913 \\
\hline Postoperatively (mean \pm SD) & $20.1 \pm 7.4$ & $43.9 \pm 16.4$ & 1.872 & $<0.001$ \\
\hline
\end{tabular}

ERAS, Enhanced Recovery After Surgery; SMD, standardized mean difference.

Table 4 Baseline characteristics before and after the 1:n propensity score match of study cohort

\begin{tabular}{|c|c|c|c|c|c|c|}
\hline Parameter & \multicolumn{3}{|c|}{ Total cohort } & \multicolumn{3}{|c|}{ PSM cohort } \\
\hline Female & $22(43.1)$ & $108(42.7)$ & 0.009 & $20(42.6)$ & $87(43.5)$ & 0.019 \\
\hline Age (years) & $62.75 \pm 15.37$ & $61.40 \pm 13.23$ & 0.094 & $62.94 \pm 15.70$ & $61.03 \pm 13.65$ & 0.130 \\
\hline $\mathrm{BMI}\left(\mathrm{kg} / \mathrm{m}^{2}\right)$ & $26.00 \pm 4.70$ & $26.11 \pm 4.87$ & 0.022 & $26.10 \pm 4.71$ & $25.84 \pm 4.82$ & 0.054 \\
\hline Neoadjuvant radiotherapy & $7(13.7)$ & $10(4.0)$ & 0.350 & $3(6.4)$ & $9(4.5)$ & 0.083 \\
\hline History of surgery (right upper abdomen) & $11(21.6)$ & $106(41.9)$ & 0.448 & $10(21.3)$ & $57(28.5)$ & 0.168 \\
\hline Preoperative serum bilirubin ( $\mathrm{mmol} / \mathrm{L}$ ) & $7.9[5.55-10.2]$ & $6.5[3.9-10.3]$ & 0.092 & $7.7[5.45-10.2]$ & $6.9[4.3-10.6]$ & 0.046 \\
\hline ASA 3-4 & $24(47.1)$ & $141(55.7)$ & 0.174 & $23(48.9)$ & $102(51.0)$ & 0.041 \\
\hline
\end{tabular}

Data are presented as mean \pm SD, median [IQR], or $n$ (\%). ERAS, Enhanced Recovery After Surgery; SMD, standardized mean difference; PSM, propensity score matching; BMI, body mass index; ASA, American Society of Anesthesiologists; IQR, interquartile range.

items (Figure 3). There were a total of 6 items that had an adherence in the ERAS cohort greater than $99 \%$ (preadmission patient education, preoperative nutritional status assessment, no oral bowel preparation, systemic opioids given, upper-body forced-air heating cover used, use of $0.9 \% \mathrm{NaCl}$, type of incision). In contrast, there were a total of 8 items that had an adherence in the ERAS cohort of less than $25 \%$ (alcohol usage, smoking behavior, epidural or spinal anaesthesia, abdominal drains, patient weight on POD1, energy intake on POD1, mobilisation at all day of surgery, mobilisation on POD1). Adherence was broken down according to the age of the patients (Figure $3 A$ ), and an age-dependent change in adherence was shown for postoperative nausea and vomiting (PONV) prophylaxis, preoperative sedative medication, and control postoperative glycemia. Adherence to individual items was not linked to ASA score (Figure 3B).

\section{Outcome measures}

The overall morbidity (Clavien-Dindo 1-5) decreased 


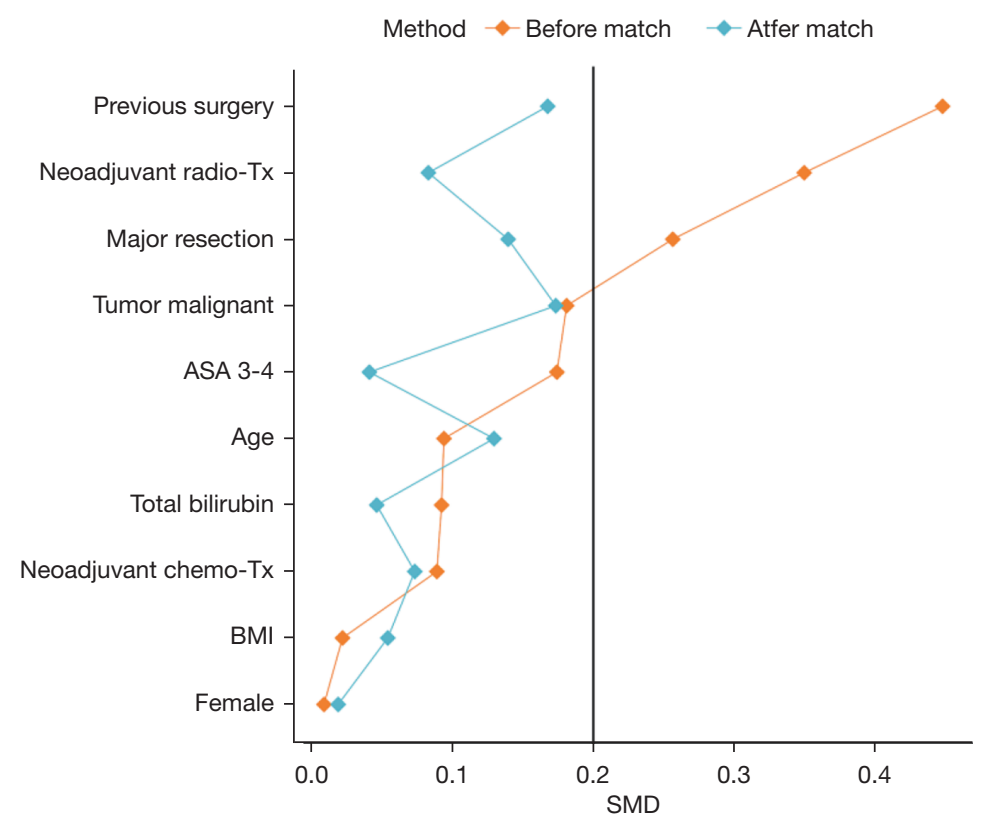

Figure 1 SMD of single parameters before and after PSM. The aim was to achieve an SMD below 0.2 indicating a balanced distribution of parameters between cohorts (Cohen's d) (14,15). ASA, American Society of Anesthesiologists; BMI, body mass index; SMD, standardized mean difference; PSM, propensity score matching.

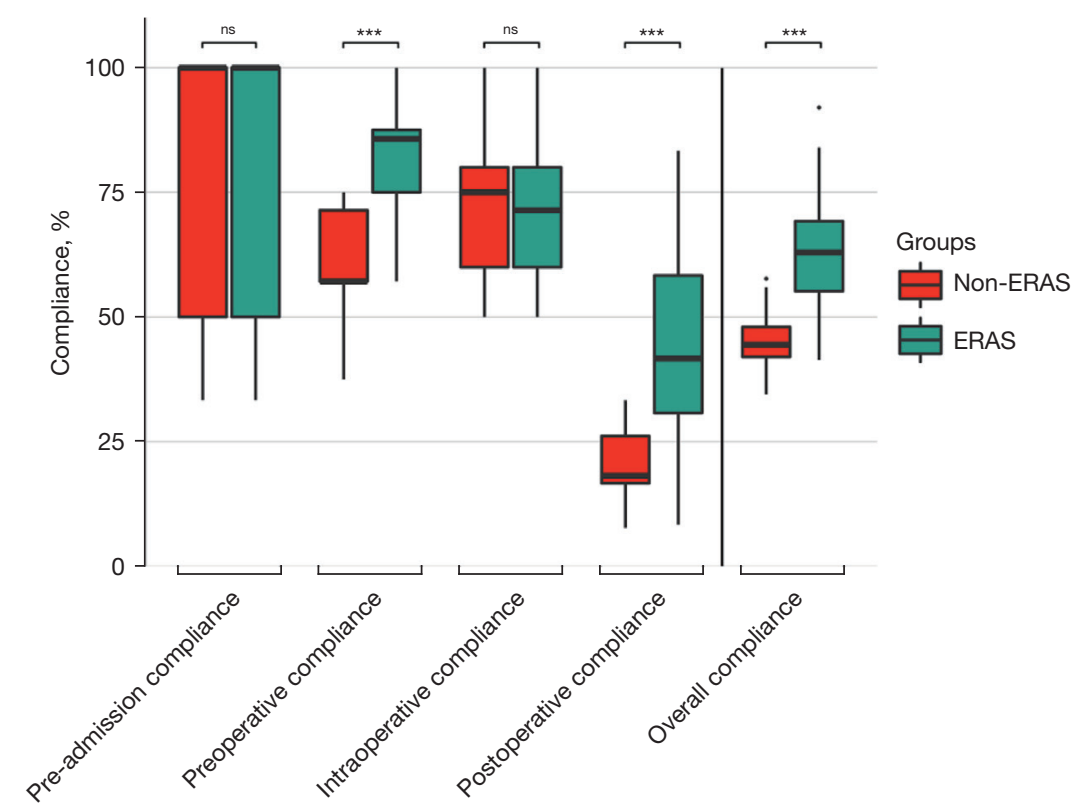

Figure 2 Adherence after $1: \mathrm{n}$ propensity score matching perioperative and overall. ***, $\mathrm{P}<0.001$, generalized estimating equation. ns, not significant; ERAS, Enhanced Recovery After Surgery. 


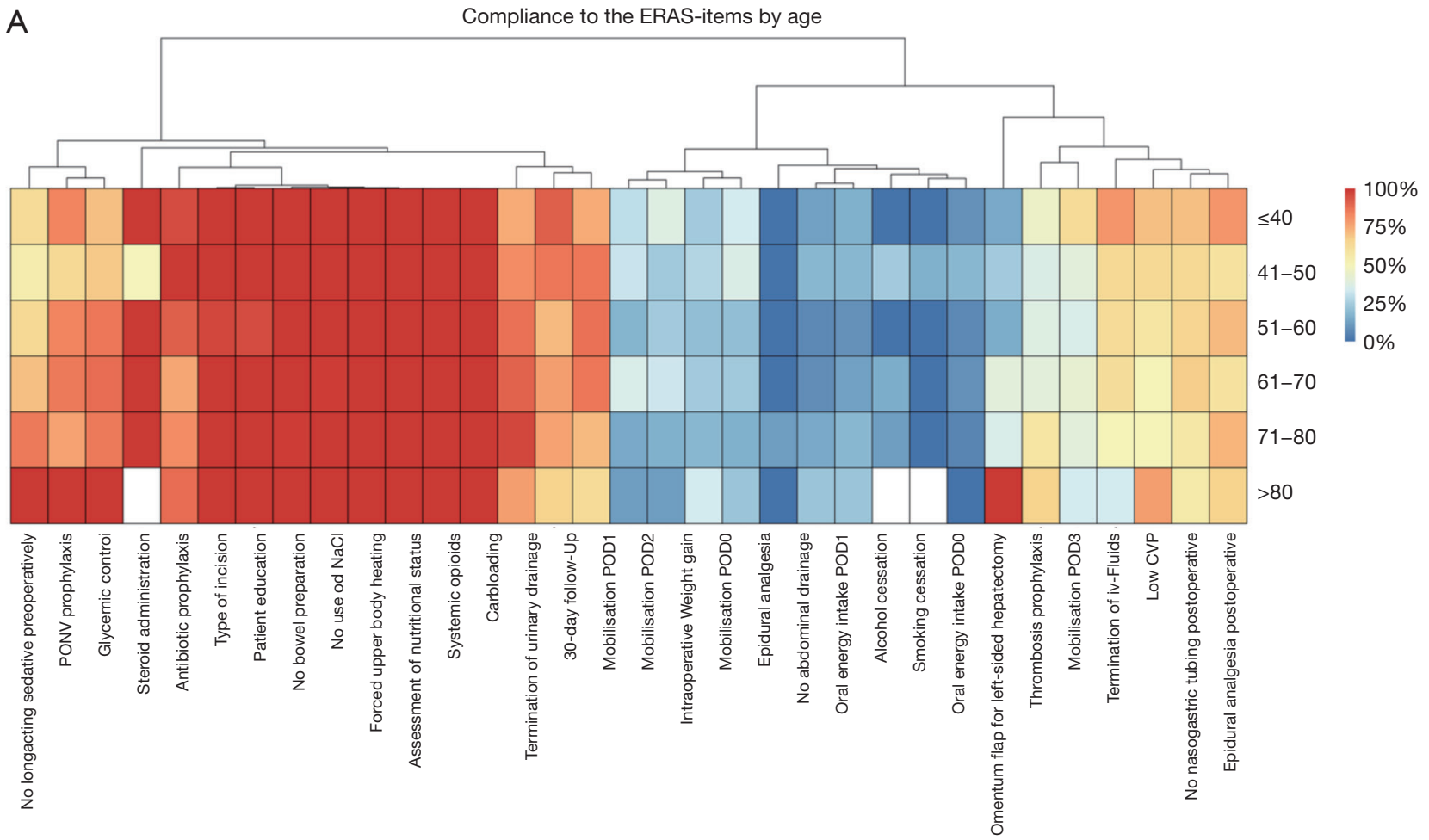

B Compliance to the ERAS-items by ASA groups
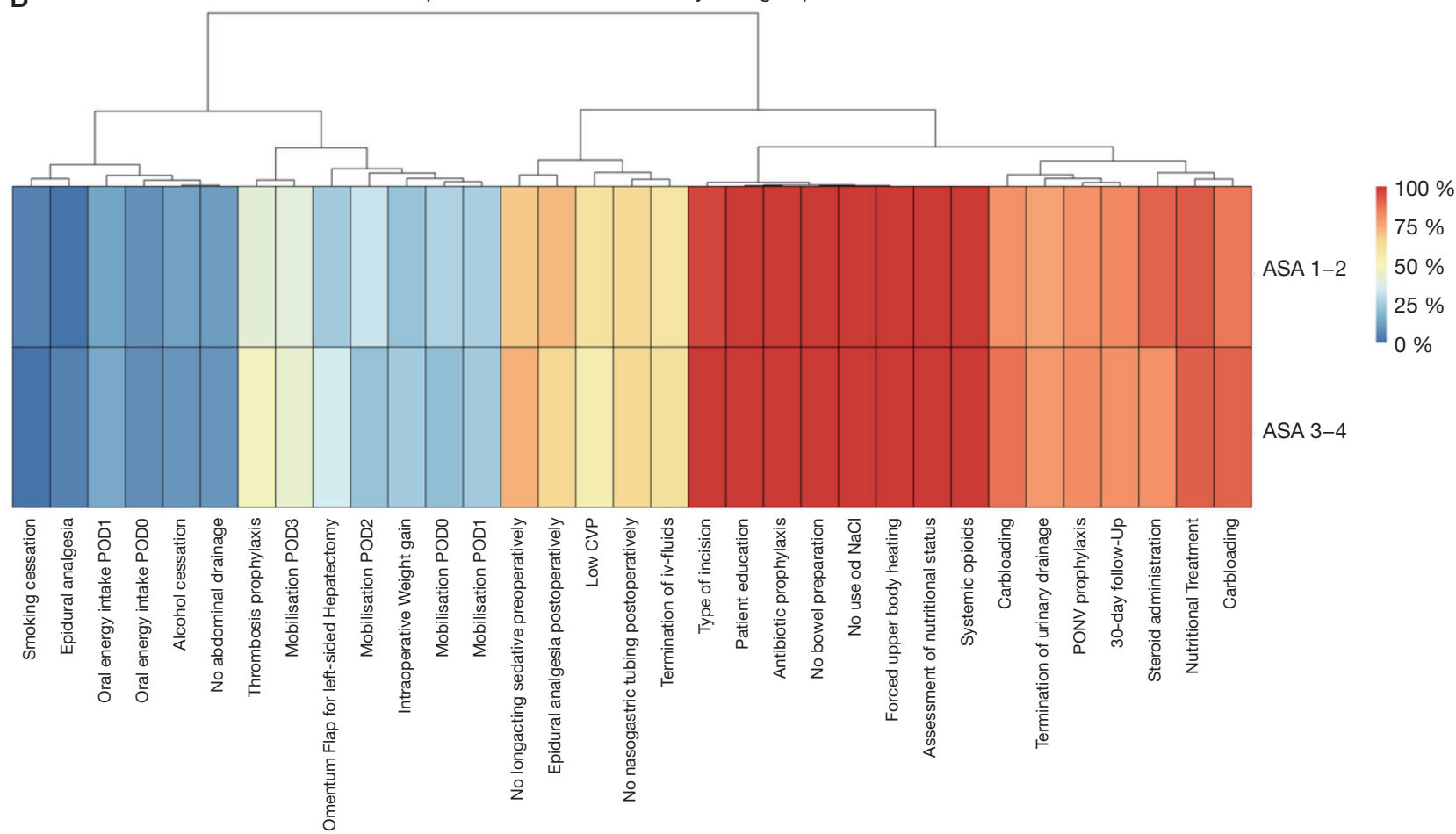

Figure 3 The level of adherence of each item ( $\mathrm{n}=31)$ were grouped by age at surgery $(\leq 40 ; 41-50 ; 51-60 ; 61-70 ; 71-80 ;>80)($ A, ERAS cohort). In addition, adherence of each item were grouped by ASA score [1-2; 3-4] (B, ERAS cohort). To display the results, a heat map was compiled by Wards' minimum variance hierarchical clustering with Euclidean distance. ERAS, Enhanced Recovery After Surgery; PONV, postoperative nausea and vomiting; POD, postoperative day; CVP, central venous pressure; ASA, American Society of Anesthesiologists. 


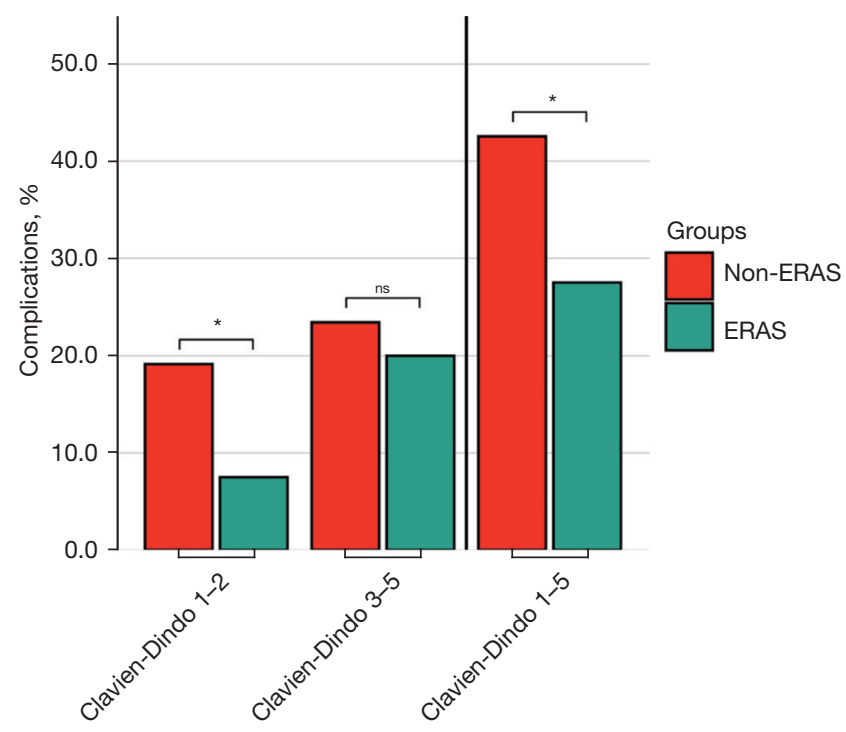

Figure 4 Complications rates after PSM (47 controls: 200 treatments) (*, $\mathrm{P}<0.05$, generalized estimating equation). ERAS, Enhanced Recovery After Surgery; ns, not significant; PSM, propensity score matching.

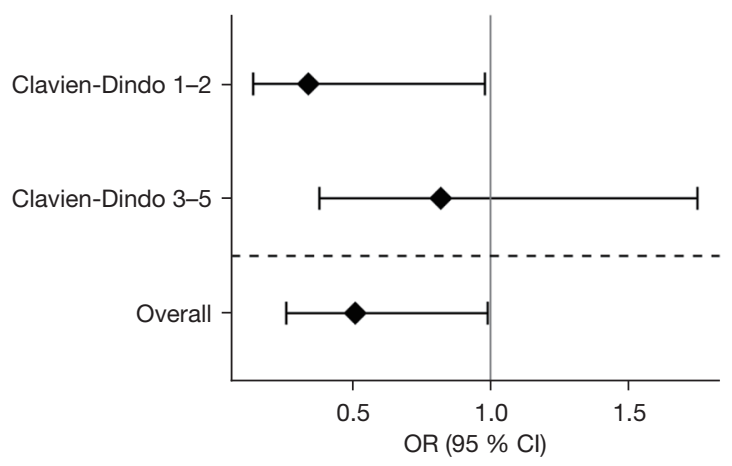

Figure 5 Odds ratios of complication rates after 1:n propensity score matching. Clavien-Dindo $1-5$ OR $=0.51$ (95\% CI: 0.26-0.99; $\mathrm{P}=0.0457$ ), Clavien-Dindo $1-2$ OR $=0.34$ (95\% CI: 0.14-0.98; $\mathrm{P}=0.0192$ ), Clavien-Dindo 3-5 OR =0.82 (95\% CI: 0.38-1.75; $\mathrm{P}=0.604)$. OR, odds ratio; $\mathrm{CI}$, confidence interval.

from $41.2 \%$ to $26.5 \%$ in the ERAS group ( $\mathrm{P}=0.042$ ). Clavien-Dindo grade 1-2 complications decreased from $17.6 \%(n=9)$ in the non-ERAS group to $7.5 \%(n=19)$ after implementing the ERAS protocol $(\mathrm{P}=0.032)$. The rate of grade $3-5$ complications was comparable at $23.5 \%(n=12)$ in the pre-ERAS group and $19.0 \%(\mathrm{n}=48)$ in the ERAS group $(\mathrm{P}=0.445$, Table 3). ICU and hospital length of stay were comparable in both groups. These results were confirmed

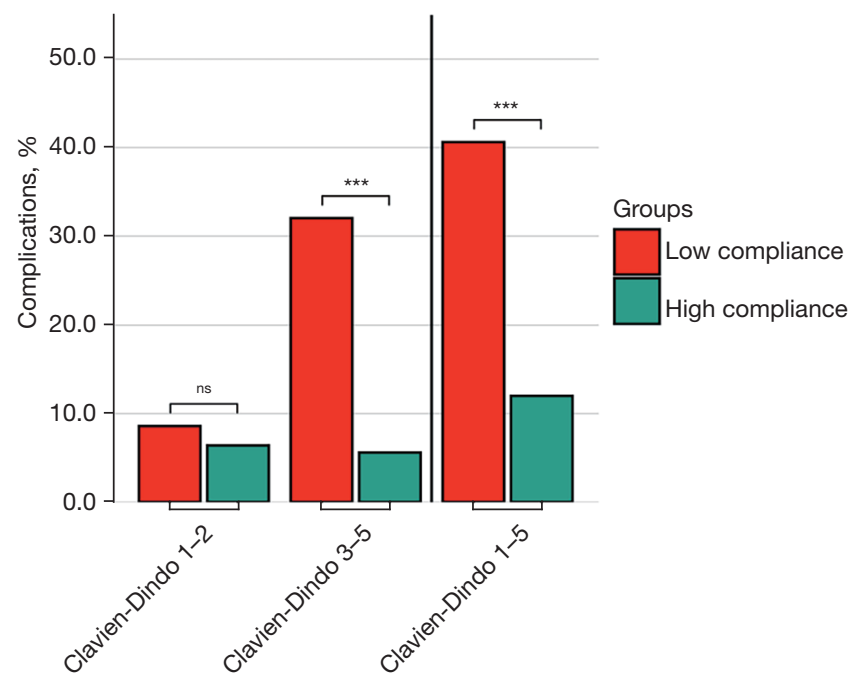

Figure 6 Complications rates by overall adherence (ERAS cohort). The median adherence among the ERAS cohort was $62.1 \%$ (the high adherence group included all with adherence $>$ median adherence; the low adherence group included all with adherence $\leq$ the median adherence). ${ }^{* * *}, \mathrm{P}<0.001$, Fisher's exact test. ns, not significant; ERAS, Enhanced Recovery After Surgery.

after PSM (Figure 4). The OR (after PSM) of developing a complication Clavien-Dindo 1-5 was 0.51 (95\% CI: 0.26-0.99; $\mathrm{P}=0.0457$ ) in the ERAS group (Figure 5), while this trend was significant for Clavien-Dindo $1-2(\mathrm{OR}=0.34$; 95\% CI: 0.14-0.98; $\mathrm{P}=0.0192)$, but not for Clavien-Dindo $3-5$ (OR $=0.82$; 95\% CI: 0.38-1.75; $\mathrm{P}=0.604)$.

The complications rates were significantly linked inversely to the adherence in the ERAS cohort (Figure 6). In the low adherence group, overall complications (ClavienDindo 1-5) were 4 times higher (40.6\%), when compared to the high adherence group $(12 \%, \mathrm{P}<0.001$, Table 5). This decrease was not present for Clavien-Dindo 1-2, while Clavien-Dindo 3-5 decreased by factor 6 in the high adherence group ( $32 \%$ vs. $5.6 \%, \mathrm{P}<0.001)$. The importance of adherence was analysed separately according to the different phases (Table 6). The ERAS protocol did not change the adherence to pre-admission and intraoperative items $(\mathrm{P}>0.05)$. In contrast, adherence was increased from $59.4 \%$ to $83.6 \%$ preoperatively $(\mathrm{P}<0.001)$ and from $20.4 \%$ to $44.2 \%$ postoperatively $(\mathrm{P}<0.001)$. Low adherence in the pre-admission group also failed to demonstrate an effect on the complication rate (Figure S1). For preoperative adherence, there was a significant reduction 
Table 5 Complication rates of ERAS cohort according to adherence

\begin{tabular}{|c|c|c|c|}
\hline Parameter & \multicolumn{2}{|c|}{ Complications rate, $\mathrm{n}(\%)$} & $\mathrm{P}$ \\
\hline Clavien-Dindo 1-5 & $52(40.6)$ & $15(12.0)$ & $<0.001$ \\
\hline Clavien-Dindo 1-2 & $11(8.6)$ & $8(6.4)$ & 0.635 \\
\hline Clavien-Dindo 3-5 & $41(32.0)$ & $7(5.6)$ & $<0.001$ \\
\hline
\end{tabular}

P: Fisher's exact test. ERAS, Enhanced Recovery After Surgery.

Table 6 Perioperative adherence according to 1:n propensity score matching

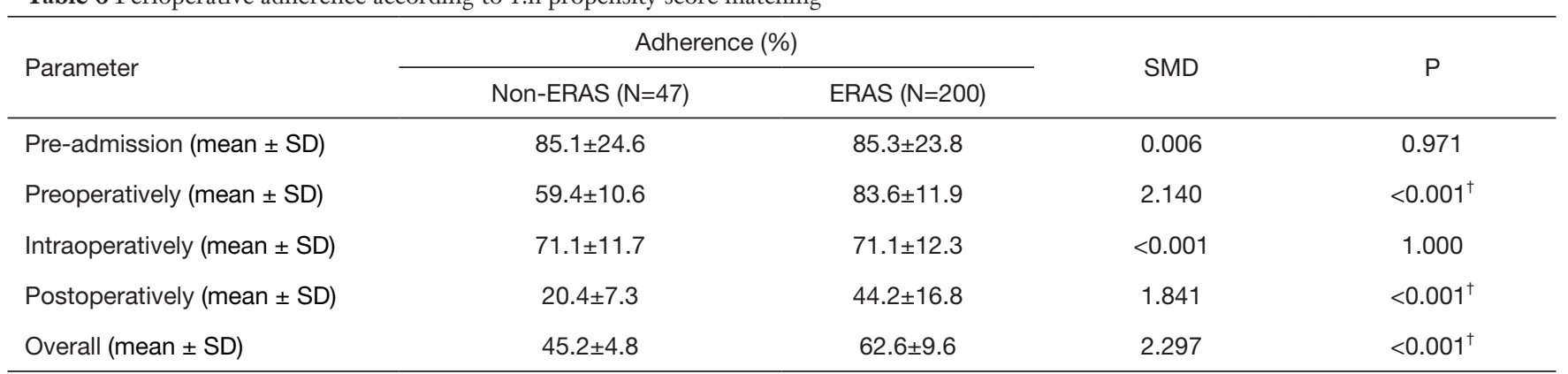

${ }^{\dagger}, \mathrm{P}<0.001$, GEE. ERAS, Enhanced Recovery After Surgery; SMD, standardized mean difference; GEE, generalized estimating equation.

in complications Clavien-Dindo 3-5 by more than half, whereas no difference was observed for Clavien-Dindo 1-2 (Figure S2). Furthermore, a highly significant reduction in complications Clavien-Dindo 3-5 for intraoperative adherence and postoperative adherence was observed (Figures S3,S4).

\section{Technical implications of the liver resection}

Next, we analyzed the potential value of the ERAS items differentiated by the surgical approach, i.e., conventional open approach or MILS including laparoscopic and roboticassisted liver resections. To make the groups comparable we applied a PSM matching for open surgery (Table S1). Adherence could be increased preoperatively and postoperatively in ERAS-patients undergoing open liver surgery, when compared to non-ERAS patients undergoing open liver surgery (Table S2). There was a trend towards less overall complications in the ERAS-group, however, being short of statistical significance $(P>0.05$, Table S3). Apparently, this was due to a (non-significant) reduction in minor complications, as the rate of major complications did not differ. Possibly, due to the small number of matched patients receiving open liver surgery, differences in complication rates were not statistically significant.
In patients undergoing MILS a significant reduction of overall complications was noted (Tables S4,S5). This can be attributed to a reduction of both minor and major complications, although statistical significance was not reached in those subclassifications $(\mathrm{P}>0.05$, Table S6).

\section{Discussion}

Implementation of the ERAS protocol reduces minor complications in liver surgery, which is particularly true for patients scheduled for minimally invasive approaches. Overall patient adherence was significantly increased by using the EIAS, especially in the preoperative and postoperative course. Basically, the rate of increase of adherence depends on the extent of adherence before ERAS implementation. Therefore, the level of adherence in the pre-ERAS group was high preoperatively, especially in the pre-admission phase, so that at this point no relevant increase occurred after ERAS implementation. Some ERAS items were easier to implement with excellent adherence $>90 \%$ (ERAS items 1, 2, 5, 7, 9, 12, 16, 18, 19). In contrast, other ERAS items were almost impossible to implement like smoking behavior or alcohol consumption (ERAS-Item 3 and 4). From our point of view, individual ERAS items need to be discussed and adapted in the context of MILS. In the 
following, we will discuss the ERAS recommendation for liver surgery, their transfer into the EIAS database with its definition, their implementation at our center, and their applicability for liver surgery (Table 1).

\section{Pre-admission (1-2 weeks before surgery):}

If the NSR was $\geq 3$, a preoperative immunonutrition was administered (perioperative oral immunonutrition, ERAS-Item 2). Immunonutrition worked well and it could be applied with adherence of $>92 \%$. An ERAS nurse was assigned to this task, which might explain the high application rate. Adherence to preoperative NSR assessment was $100 \%$, and adherence to food administration was $92 \%$ (ERAS-Items 1-2). In contrast, for other ERAS items it was difficult to reach high adherence like smoking behavior (ERAS-Item 3) or alcohol consumption (ERASItem 4) demonstrating an adherence of $11.1 \%$ and $1.9 \%$, respectively. It appears obviously that lifestyle changes and substance abuse is difficult to address by an ERAS program and might be beyond the scope of such guidelines.

\section{Preoperatively (1 day and bours before surgery)}

Preoperative education item showed a high adherence $>99 \%$ in the ERAS cohort and worked well (ERASItem 5). We regularly performed a patient information and motivation talk by ERAS nurses, handed out patient information materials and a patient diary. No patient should receive mechanical preoperative bowel preparation and this item seems reasonable (adherence of $>99 \%$, ERASItem 7). A carbohydrate-rich sip feed was administered at $10 \mathrm{pm}$ the day before surgery and 2 hours before the onset of anesthesia and good adherence of $88 \%$ was achieved (preoperative fasting and preoperative carbohydrate load, ERAS-Item 6). The avoidance of preoperative long-acting sedative medication was achieved in $71 \%$, while apparently adherence increased with increasing age (Figure $3 \mathrm{~A}$, ERAS-Item 8). This might be explained by the fact of a higher rate of well informed patients with younger age, who explicitly demand sedative medication (16). Perioperative thromboprophylaxis (combined mechanical and pharmacological) was reduced with an overall $44 \%$ adherence Antithrombotic prophylaxis, ERAS-Item 10). The reduced value was due to the lack of preoperative administration of $\mathrm{LMWH}$ and difficulties in implementing routine procedures in the normal ward as part of such a program. In this regard, there is certainly still a need for action at our center. However, we did not notice any thrombotic events since implementing ERAS. Adherence to perioperative steroid administration was $85 \%$, although this should not be used in patients with diabetes. However, this information and awareness is not always available during surgery, which is why some patients with diabetes received steroids. Note, this recommendation has been given a weak grade according to the guidelines from 2016 (2), while a recent double-blind randomized controlled trial (RCT) was currently able to prove that a single preoperative dose of methylprednisolone significantly reduces length of hospital stay and complications following major hepatectomy (17). Antibiotic prophylaxis before incision was easy to apply and was already established before implementation of the ERAS program (adherence $>99 \%$, ERAS-Item 9). Skin preparation as recommended by the guidelines was not applied in our program, since this was not covered by EIAS of the ERAS society. However, the internal standard in our department is topical application of alcohol-based disinfectant (Kodan, Schülke \& Mayr GmbH, Norderstedt, Germany).

\section{Intraoperatively}

A Mercedes-like incision was avoided whenever possible, and adherence was thus $98 \%$ (ERAS-Item 12). EIAS did not introduce an adherence item for MILS because there are still resections that are not routinely performed minimally invasively, especially in case of vascular, biliary reconstruction or excessive adhesions due to previous surgery (9). However, a high percentage of MILS including robotic-assisted resections were achieved with $69.6 \%$ in our ERAS cohort. The nasogastric tube should be removed at the end of surgery (ERAS-Item 20). However, some patients display decreased vigilance after liver resection. In these cases, the tube is maintained until full recovery of vigilance to prevent aspiration. Furthermore, we believe that a tube may well be justified for 1-2 days after major hepatectomy with biliary reconstruction and resulting gastroparesis. Therefore, our adherence of only $64 \%$ can be explained. An adherence close to $100 \%$ seems unlikely at centers with a high percentage of extended resections and biliary reconstructions. We would recommend a more differentiated approach for this item. The placement of abdominal drains in liver surgery is controversial (ERASItem 13) $(18,19)$ and the recommendations at this point are stated as weak (2). In principle, drainage seems to be rational, especially when biliary reconstructions and major liver resections are performed. Although several 
high quality trials exist, they did not include representative cohorts of patients with extended liver resections (8). In our cohort, an adherence of $10.6 \%$ adherence rate was achieved. As with nasogastric tubes, we consider a stratified approach to be more rational, differentiating between low and highrisk patients. Preventing intraoperative hyperthermia was implemented without difficulties and adherence in the ERAS cohort was $100 \%$ (ERAS-Item 18). Of note, this is standard of care at our center, and has been implemented before ERAS. Omentum flap was performed in only 29\% of patients with left-sided hepatectomy and in none of the patients undergoing minimally invasive left-sided liver resection (ERAS-Item 14). Omentum flap has shown to be beneficial for reducing delayed gastric emptying in RCTs (with small numbers of patients, $n=40$ and $n=21)(20,21)$. It remains questionable whether this item is transferable into real-life surgery, since the use of MILS increases and a flap is technically hardly possible in every case (e.g., history of colorectal surgery, colorectal liver metastasis, redo-resection). When at risk for PONV (Apfel score ${ }^{2} \geq 2$ ), multimodal PONV prophylaxis (drug and nondrug) was administered with an adherence of $81.3 \%$ (ERAS-Item 15). The volume of crystalloid and colloid infusions and of blood products, as well as central venous pressure (CVP) immediately before parenchymal transection, was recorded (ERAS-Items 19, 21, 22, 23). A CVP below 5 could be achieved in only $56 \%$ of ERAS patients. Even though there is high expertise at our center with up to 400 liver resections per year, this item is important but not always achievable, especially in MILS. Of note, the CVP is incorrect during MILS based on positioning alone. A widely accepted equation to account for pressure of the pneumoperitoneum could be helpful to find individual thresholds. The adherence to not using $0.9 \% \mathrm{NaCl}$ was achieved in $100 \%$ of all ERAS patients.

\section{Postoperatively}

According to the EIAS, all patients should have at least one packet of oral sip-feed with an energy content of $300 \mathrm{kcal}$ each up to 4 hours postoperatively on day of surgery (ERASItems 24-25). On POD1, two packets of oral sip-feed should be consumed $(600 \mathrm{kcal})$. The adherence on POD0 and POD1 was low with $7 \%$ and $14 \%$, respectively. The reasons remain vague, but might be explained by observations of patients disliking the over-sweetened sip-feeds. Some patients even had to throw up. It should be noted that the ERAS guideline recommends oral sip-feeds only for malnourished patients, as most patients can eat normal food following liver surgery (2). The termination of i.v. fluid after $\leq 1$ night was only possible in $60.1 \%$ of the patients. Fluid management remains individual and has to be modified, whenever necessary. One of the most challenging ERAS items is early mobilization (Early mobilization, 26-30). The ERAS guidelines for liver surgery point out that early mobilization after liver resection should be encouraged from the morning after the operation until hospital discharge (evidence level: low, grade of recommendation: weak). The transfer of this recommendation into the EIAS database resulted in 4 ERAS items: (I) mobilization on the day of surgery; (II) mobilization on POD1; (III) mobilization on POD2; and (IV) mobilization on POD3. For reaching the $100 \%$ adherence status of this ERAS item, the patient should have any mobilization outside the bed (time period not important), on POD1 the patient should be mobilized $>4 \mathrm{~h}$, on POD $2>6 \mathrm{~h}$ and on POD $3>6$ h. The duration of mobilization on POD1-3 was documented in hours as well as the duration until preoperative mobility was achieved. The adherence for these items ranged from $22 \%$ to $41 \%$ increasing from dayto-day after surgery. Early mobilization can be pursued with the support of nurses and physiotherapists, although it may not always be achieved depending on performance status. This accounts especially for complex liver resections. This item is important, but adherence is sharply linked to the extent of liver resection, length of surgery, motivation of the patient and performance status. It should also be noted that the daily thresholds of hours mobilized required by the EIAS were derived from colorectal surgery and have not yet been confirmed in liver surgery so far (2) (EIAS-Compliance Calculation, Guide Liver v4.5.2.1). Postoperative glycaemic control was achieved in $84 \%$ in the ERAS cohort and the item is well applicable (ERASItem 31). Stimulation of bowel movement: This item is not included in the adherence calculation by EIAS of the ERAS society. The gastrointestinal tract stimulation performed (laxatives and/or chewing gum) and the documentation of duration until the first flatus, stool and until the first full meal is well established in our department. This might be applicable for all surgical liver centers. No or only shortacting opioids (fentanyl, remifentanil, sufentanil) should be administered intraoperatively and we achieved adherence of $100 \%$ (ERAS-Items 16, 17, 32). The guidelines for liver surgery pointed out peridural anesthesia (PDA) should be omitted for open liver resections because of possible kidney failure due to hypotension (2). Our ERAS cohort showed an 
overall adherence of $2 \%$ according to the EIAS adherence calculation, while $67.5 \%$ of patients undergoing open liver resection did not receive any PDA. Patients who did not receive PDA for "other reasons" ( $65.5 \%$ in our study) were rated as non-compliant, while EIAS only considers patients to be compliant in whom the PDA is not used due to it being "contraindicated". We do not agree with this distinction/classification, as it only seems relevant whether a PDA has been applied or not. Audits are recommended in the ERAS guidelines but are not part of the EIAS. We held biweekly ERAS team meetings with analyses of the current status of ERAS implementation and obstacles to be overcome in the process helped to increase adherence and decrease complication rates over time.

Adherence is one of the key factors for the successful implementation of an ERAS protocol. Detailed adherence data for the ERAS Guidelines for Liver Surgery do not exist to date and this study is therefore the first study to report on the adherence for each perioperative phase and specific ERAS item $(2,8,22,23)$. Individual items of the guidelines have been validated whether they are suitable for liver surgery. Some items showed reduced adherence such as alcohol consumption with $1.9 \%$, and others a very high with $100 \%$ such as nutritional status. The protocol has to be improved considering our findings. In a 2020 meta-analysis, Noba and colleagues included 6 RCTs and 21 cohort studies involving 3,739 patients undergoing liver surgery with modified ERAS protocols not identical to the guidelines (8). Interestingly, only 4 out of 27 studies (474 patients) provided data on adherence with ERAS elements. Three of the four studies measured overall compliance with the ERAS elements. Adherence was higher in the ERAS group in all three studies, ranging from $65 \%$ to $73.8 \%$ in the ERAS group and $20 \%$ to $48.7 \%$ in the standard group. Indeed, we were able to achieve adherence for pre-admission $86.9 \%$, for preoperative $83.8 \%$ and for intraoperative $71.6 \%$. These values are within our range. One randomised control trial by Jones et al. (46 patients ERAS and 45 patient non-ERAS) reported adherence $100 \%$ for 18 of the 19 ERAS items in the ERAS group (24). Based on these numbers, a problem with the definition of the adherence of each ERAS item becomes apparent. For example, is a patient early mobilized if they get up and walk around for a few minutes on the first POD to achieve $100 \%$ adherence, or does the patient achieve full adherence if they get up, walk around, and sit in the chair for more than 4 hours. Thus, achieving adherence depends on the exact definition of the items. This must therefore be taken into account in future analysis of work with ERAS adherence rates.

Interestingly, we were able to further reduce the postoperative complication rate by introducing all ERAS items according to the ERAS society guidelines of liver surgery, albeit our center is highly specialised for liver resections (25). This reduction was achieved due to a decreased rate of minor complications (Clavien-Dindo 1 and 2) rather than major complications (Clavien-Dindo 3-5). There are already other studies showing a positive effect of ERAS on postoperative complication rates after liver resection (8). However, none of these studies analysed the ERAS guidelines for liver surgery from the ERAS society and questioned the adherence of the proposed items. Nevertheless, the present study is limited as well by its observational character and a randomization of higher evidence would be desirable.

There was no difference of length of stay between the non-ERAS (7 days; range, 5.5-9 days) and the ERAS patients (7 days; range 5-9 days). The causality that the ERAS program had no effect here is not clear, although the length of stay is already low and consistent with that of other MILS programs, as shown by a large meta-analysis for ERAS protocols in liver surgery (26). It is important to note that health insurance companies in Germany set a threshold length of stay for certain procedures and reimbursement is tied to this.

\section{Conclusions}

Implementation of the ERAS protocol for liver surgery according to the ERAS guidelines of the ERAS society reduces Clavien-Dindo grade 1-2 complications in our cohort particularly in patients who underwent MILS. Overall patient adherence was significantly increased by using the EIAS. However, individual ERAS items need to be discussed and adapted due to lack of adherence definition and practicality especially in MILS.

\section{Acknowledgments}

Funding: None.

\section{Footnote}

Reporting Checklist: The authors have completed the STROBE reporting checklist. Available at https://hbsn. amegroups.com/article/view/10.21037/hbsn-21-294/rc 
Data Sharing Statement: Available at https://hbsn. amegroups.com/article/view/10.21037/hbsn-21-294/dss

Conflicts of Interest: All authors have completed the ICMJE uniform disclosure form (available at https://hbsn. amegroups.com/article/view/10.21037/hbsn-21-294/coif). The authors have no conflicts of interest to declare.

Ethical Statement: The authors are accountable for all aspects of the work in ensuring that questions related to the accuracy or integrity of any part of the work are appropriately investigated and resolved. The study was conducted in accordance with the Declaration of Helsinki (as revised in 2013). This prospective observational cohort study was approved by the local ethics committee of the Charité-Universitätsmedizin Berlin under the registration numbers EA2/108/18 and EA4/153/18, and was registered with the German Clinical Trials Register (No. DRKS00011631). All study participants gave written informed consent for study inclusion.

Open Access Statement: This is an Open Access article distributed in accordance with the Creative Commons Attribution-NonCommercial-NoDerivs 4.0 International License (CC BY-NC-ND 4.0), which permits the noncommercial replication and distribution of the article with the strict proviso that no changes or edits are made and the original work is properly cited (including links to both the formal publication through the relevant DOI and the license). See: https://creativecommons.org/licenses/by-nc-nd/4.0/.

\section{References}

1. Ljungqvist O, Scott M, Fearon KC. Enhanced Recovery After Surgery: A Review. JAMA Surg 2017;152:292-8.

2. Melloul E, Hübner M, Scott M, et al. Guidelines for Perioperative Care for Liver Surgery: Enhanced Recovery After Surgery (ERAS) Society Recommendations. World J Surg 2016;40:2425-40.

3. Ren QP, Luo YL, Xiao FM, et al. Effect of enhanced recovery after surgery program on patient-reported outcomes and function recovery in patients undergoing liver resection for hepatocellular carcinoma. Medicine (Baltimore) 2020;99:e20062.

4. Zhou JM, He XG, Wang M, et al. Enhanced recovery after surgery program in the patients undergoing hepatectomy for benign liver lesions. Hepatobiliary Pancreat Dis Int 2020;19:122-8.
5. Jing X, Zhang B, Xing S, et al. Cost-benefit analysis of enhanced recovery after hepatectomy in Chinese Han population. Medicine (Baltimore) 2018;97:e11957.

6. Qi S, Chen G, Cao P, et al. Safety and efficacy of enhanced recovery after surgery (ERAS) programs in patients undergoing hepatectomy: A prospective randomized controlled trial. J Clin Lab Anal 2018;32:e22434.

7. Liang $X$, Ying $H$, Wang $H$, et al. Enhanced recovery care versus traditional care after laparoscopic liver resections: a randomized controlled trial. Surg Endosc 2018;32:2746-57.

8. Noba L, Rodgers S, Chandler C, et al. Enhanced Recovery After Surgery (ERAS) Reduces Hospital Costs and Improve Clinical Outcomes in Liver Surgery: a Systematic Review and Meta-Analysis. J Gastrointest Surg 2020;24:918-32.

9. Schmelzle M, Krenzien F, Schöning W, et al. Laparoscopic liver resection: indications, limitations, and economic aspects. Langenbecks Arch Surg 2020;405:725-35.

10. Schmelzle M, Krenzien F, Schöning W, et al. Possibilities and limits of robotic liver surgery - Current status 2020. Chirurg. Available online: https://doi.org/10.1007/s00104020-01300-w

11. Krenzien F, Nevermann N, Krombholz A, et al. Treatment of Intrahepatic Cholangiocarcinoma-A Multidisciplinary Approach. Cancers 2022;14:362.

12. von Elm E, Altman DG, Egger M, et al. The Strengthening the Reporting of Observational Studies in Epidemiology (STROBE) statement: guidelines for reporting observational studies. J Clin Epidemiol 2008;61:344-9.

13. Kondrup J, Allison SP, Elia M, et al. ESPEN guidelines for nutrition screening 2002. Clin Nutr 2003;22:415-21.

14. Cohen J. Statistical Power Analysis for the Behavioral Sciences. Routledge; 2013.

15. Schuler MS, Chu W, Coffman D. Propensity score weighting for a continuous exposure with multilevel data. Health Serv Outcomes Res Methodol 2016;16:271-92.

16. Johansson V, Islind AS, Lindroth T, et al. Online Communities as a Driver for Patient Empowerment: Systematic Review. J Med Internet Res 2021;23:e19910.

17. Bressan AK, Isherwood S, Bathe OF, et al. Preoperative Single-dose Methylprednisolone Prevents Surgical Site Infections After Major Liver Resection: A Randomized Controlled Trial. Ann Surg 2022;275:281-7.

18. Globke B, Schmelzle M, Bahra M, et al. Drainages in abdominal surgery: (in)dispensable? Chirurg 2017;88:395-400. 
19. Anweier N, Apaer S, Zeng Q, et al. Is routine abdominal drainage necessary for patients undergoing elective hepatectomy? A protocol for systematic review and metaanalysis. Medicine (Baltimore) 2021;100:e24689.

20. Igami T, Nishio H, Ebata T, et al. Using the greater omental flap to cover the cut surface of the liver for prevention of delayed gastric emptying after left-sided hepatobiliary resection: a prospective randomized controlled trial. J Hepatobiliary Pancreat Sci 2011;18:176-83.

21. Yoshida H, Mamada Y, Taniai N, et al. Fixation of the greater omentum for prevention of delayed gastric emptying after left-sided hepatectomy: a randomized controlled trial. Hepatogastroenterology 2005;52:1334-7.

22. Wang C, Zheng G, Zhang W, et al. Enhanced Recovery after Surgery Programs for Liver Resection: a Meta-

Cite this article as: Schmelzle M, Krenzien F, Dahlke P, Krombholz A, Nevermann N, Feldbrügge L, Winter A, Schöning W, Benzing C, Pratschke J, Neudecker J. Validation of the Enhanced Recovery after Surgery (ERAS) society recommendations for liver surgery: a prospective, observational study. HepatoBiliary Surg Nutr 2023;12(1):20-36. doi: 10.21037/ hbsn-21-294 analysis. J Gastrointest Surg 2017;21:472-86.

23. Pickens RC, Cochran AR, Lyman WB, et al. Impact of Multidisciplinary Audit of Enhanced Recovery After Surgery (ERAS)® Programs at a Single Institution. World J Surg 2021;45:23-32.

24. Jones C, Kelliher L, Dickinson M, et al. Randomized clinical trial on enhanced recovery versus standard care following open liver resection. Br J Surg 2013;100:1015-24.

25. Krenzien F, Wabitsch S, Haber P, et al. Validity of the Iwate criteria for patients with hepatocellular carcinoma undergoing minimally invasive liver resection. J Hepatobiliary Pancreat Sci 2018;25:403-11.

26. Zhang L, Yuan Q, Xu Y, et al. Comparative clinical outcomes of robot-assisted liver resection versus laparoscopic liver resection: A meta-analysis. PLoS One 2020;15:e0240593. 


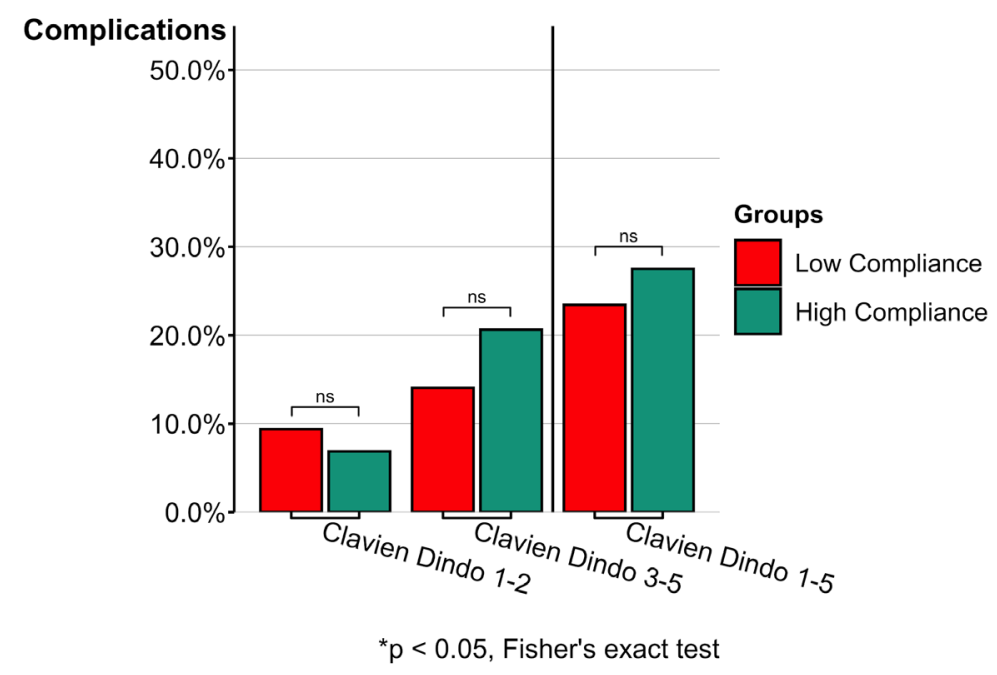

Figure S1 Complication rates by pre-admission adherence (ERAS cohort). The median adherence among the ERAS cohort was $100 \%$ (The high adherence group included all with adherence $>$ median adherence; the low adherence group included all with adherence $\leq$ the median adherence). ( ${ }^{* * *} \mathrm{P}<0.001$, Fisher's exact test).

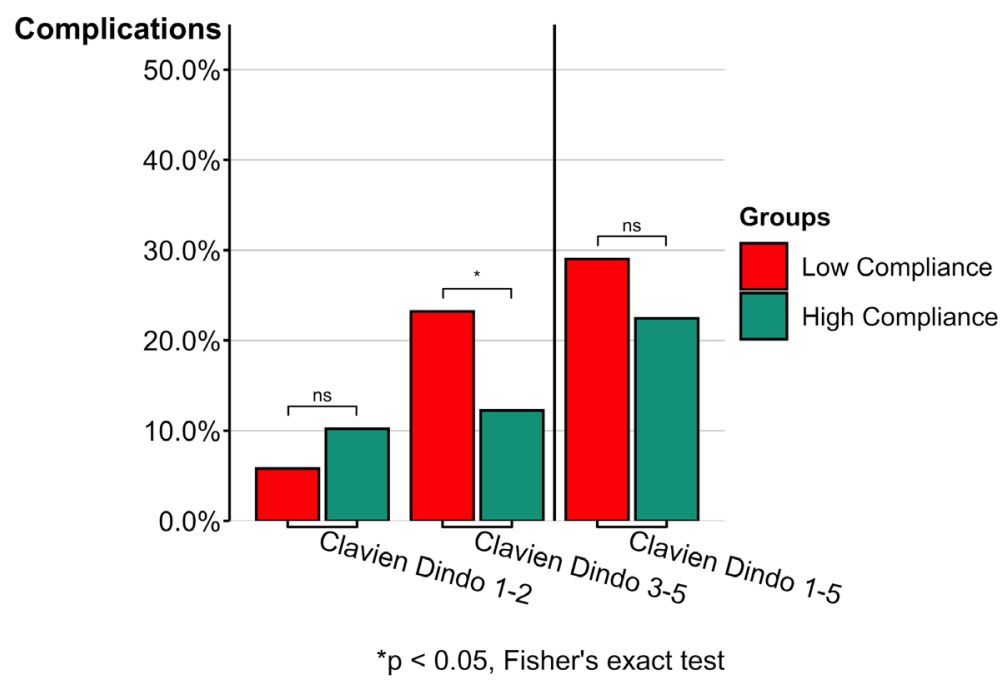

Figure S2 Complication rates by pre-operative adherence (ERAS cohort). The median adherence among the ERAS cohort was $100 \%$ (The high adherence group included all with adherence $>$ median adherence; the low adherence group included all with adherence $\leq$ the median adherence). ( ${ }^{* * *} \mathrm{P}<0.001$, Fisher's exact test). 


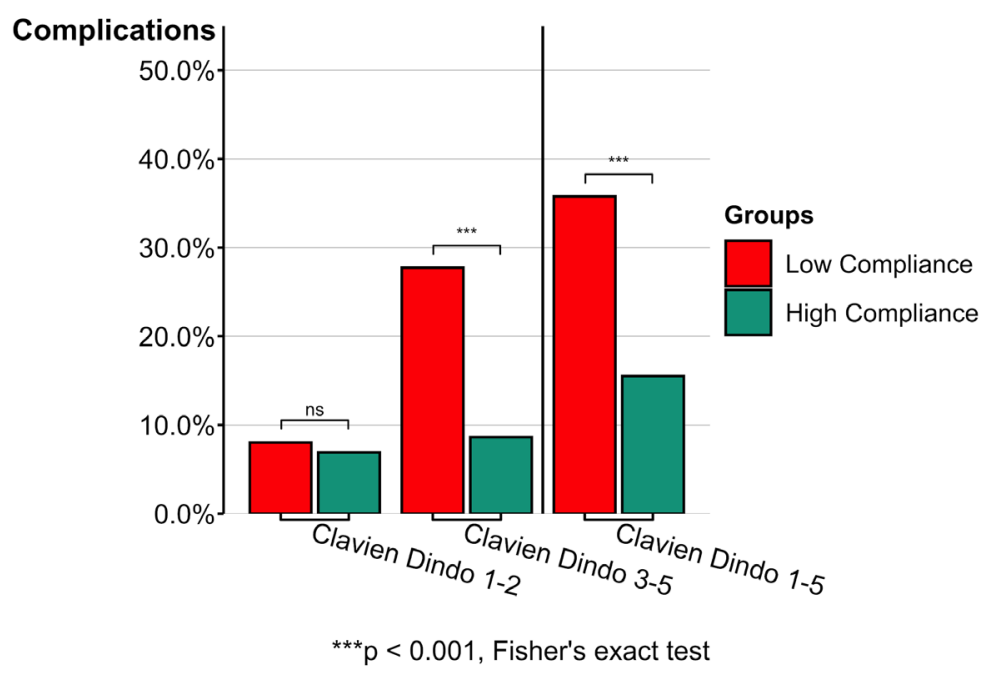

Figure S3 Complication rates by intraoperative adherence (ERAS cohort). The median adherence among the ERAS cohort was 71,4 \% (The high adherence group included all with adherence $>$ median adherence; the low adherence group included all with adherence $\leq$ the median adherence). ( ${ }^{* *} \mathrm{P}<0.001$, Fisher's exact test).

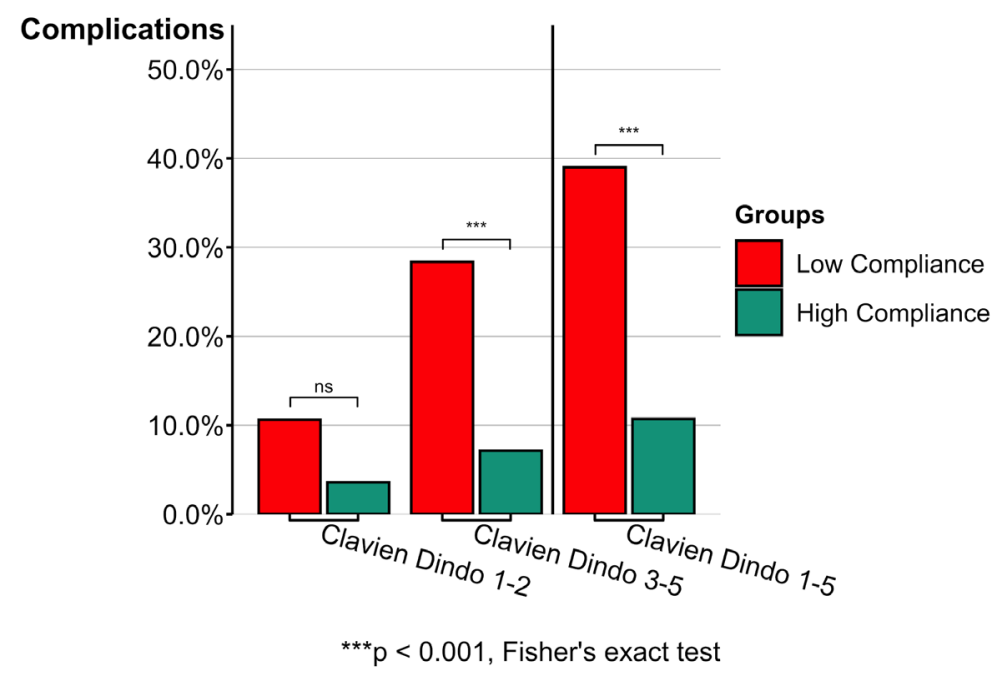

Figure S4 Complication rates by postoperative adherence (ERAS cohort). The median adherence among the ERAS cohort was $41,7 \%$ (The high adherence group included all with adherence $>$ median adherence; the low adherence group included all with adherence $\leq$ the median adherence). ( ${ }^{* * *} \mathrm{P}<0.001$, Fisher's exact test). 
Table S1 Baseline characteristics before and after the 1:n propensity score match of open surgery cohort. Mean ( \pm SD), median [IQR], or N (\%)

\begin{tabular}{|c|c|c|c|c|c|c|}
\hline & \multicolumn{3}{|c|}{ Open surgery cohort before PSM } & \multicolumn{3}{|c|}{ Open surgery cohort after PSM } \\
\hline Female & $9(52.9 \%)$ & $28(36.4 \%)$ & 0,338 & $6(50.0 \%)$ & $15(45.5 \%)$ & 0.091 \\
\hline Age (years) & $67.71( \pm 11.97)$ & $62.42( \pm 12.66)$ & 0.429 & $64.67( \pm 13.14)$ & $62.30( \pm 13.7)$ & 0.176 \\
\hline $\mathrm{BMI}\left(\mathrm{kg} / \mathrm{m}^{2}\right)$ & $26.06( \pm 4.03)$ & $26.38( \pm 5.10)$ & 0.070 & $26.41( \pm 4.37)$ & $26.21( \pm 5.05)$ & 0.043 \\
\hline Neoadjuvant radiotherapy & $1(5.9 \%)$ & $4(5.2 \%)$ & 0.030 & $1(8.3 \%)$ & $2(6.1 \%)$ & 0.088 \\
\hline History of surgery (right upper abdomen) & $7(41.2 \%)$ & $34(44.2 \%)$ & 0.060 & $5(41.7 \%)$ & $13(39.4 \%)$ & 0,046 \\
\hline Preoperative serum bilirubin (mmol/L) & $8.4[5.6-10.8]$ & $8.6[5.5-13.2]$ & 0.018 & $8.75[5.9-13.6]$ & $9.2[5.3-14]$ & 0.098 \\
\hline ASA 3-4 & $8(47.1 \%)$ & $45(58.4 \%)$ & 0.229 & $6(50.0 \%)$ & $16(48.5 \%)$ & 0.030 \\
\hline
\end{tabular}

ASA, American Society of Anesthesiologists; BMI, Body Mass Index; SMD, Standardized Mean Difference.

Table S2 Adherence in the perioperative phases and overall according to 1:n propensity score match of open surgery cohort

\begin{tabular}{llcc}
\hline Parameter & Non-ERAS $(\mathrm{N}=12)$ & ERAS $(\mathrm{N}=33)$ & $P$ \\
\hline Adherence & & & \\
pre-admission & $81.9 \%( \pm 27.0 \%)$ & $90.9 \%( \pm 20.0 \%)$ & $<.275$ \\
preoperatively & $62.2 \%( \pm 13.3 \%)$ & $84.5 \%( \pm 11.7 \%)$ & $0.001^{\star \star *}$ \\
intraoperatively & $64.6 \%( \pm 10.7 \%)$ & $64.5 \%( \pm 8.3 \%)$ & 0.980 \\
postoperatively & $18.3 \%( \pm 8.1 \%)$ & $34.8 \%( \pm 13.7 \%)$ & $<0.001^{* * *}$ \\
overall & $44.3 \%( \pm 5.1 \%)$ & $57.4 \%( \pm 8.1 \%)$ & $<0.001^{* * *}$ \\
\hline
\end{tabular}

SMD, Standardized Mean Difference, ${ }^{\dagger} \mathrm{P}<0.001$, Generalized Estimating Equation (GEE).

Table S3 Complications rate after 1:n propensity score match of open surgery cohort. (12 Controls : 33 Treatments)

\begin{tabular}{lccc}
\hline Parameter & Non-ERAS $(\mathrm{N}=12)$ & ERAS $(\mathrm{N}=33)$ & $\mathrm{P}$ \\
\hline Complication rate & & & \\
Clavien-Dindo $1-5$ & $8(66.7 \%)$ & $15(45.5 \%)$ & 0,214 \\
Clavien-Dindo $1-2$ & $4(33.3 \%)$ & $4(12.1 \%)$ & 0,113 \\
Clavien-Dindo 3-5 & $4(33.3 \%)$ & $11(33.3 \%)$ & 1 \\
\hline
\end{tabular}

${ }^{*} \mathrm{P}<0.05$, Generalized Estimating Equation (GEE). 
Table S4 Baseline characteristics before and after the 1:n propensity score match of minimal-invasive cohort. Mean ( \pm SD), median [IQR], or N (\%)

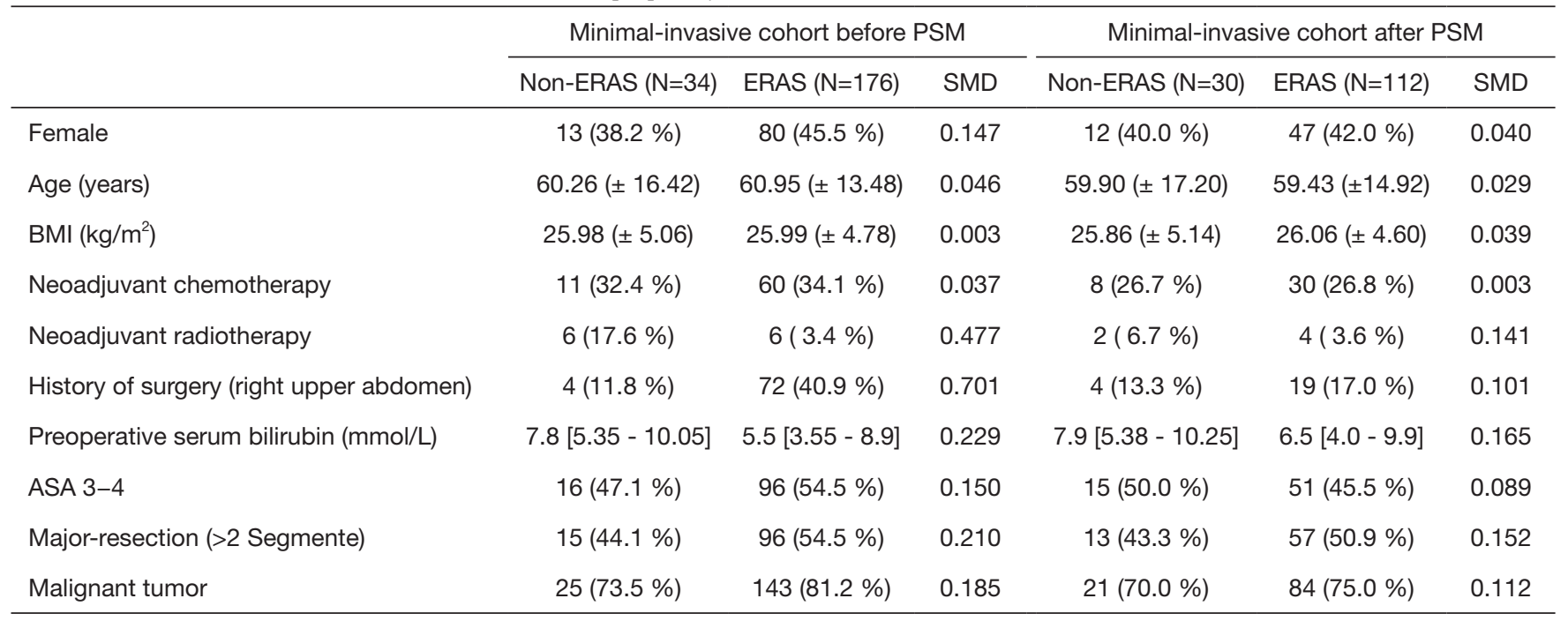

ASA, American Society of Anesthesiologists; BMI, Body Mass Index; SMD, Standardized Mean Difference.

Table S5 Complications rate after 1:n propensity score match of the minimal-invasive cohort. (30 Controls : 112 Treatments)

\begin{tabular}{lccc}
\hline Parameter & Non-ERAS $(\mathrm{N}=30)$ & ERAS $(\mathrm{N}=112)$ & $P$ \\
\hline Complication rate & & & $0.036^{\star}$ \\
Clavien-Dindo 1-5 & $9(30.0 \%)$ & $15(13.4 \%)$ & 0.054 \\
Clavien-Dindo 1-2 & $413.3 \%)$ & $4(3.6 \%)$ & 0.298 \\
Clavien-Dindo 3-5 & $4(13.3 \%)$ & $11(9.8 \%)$ & \\
\hline
\end{tabular}

${ }^{*} \mathrm{P}<0.05$, Generalized Estimating Equation (GEE).

Table S6 Adherence in the perioperative phases and overall according to 1:n propensity score match of the minimal-invasive cohort

\begin{tabular}{lccr}
\hline Parameter & Non-ERAS $(\mathrm{N}=12)$ & ERAS $(\mathrm{N}=33)$ & $P$ \\
\hline Adherence & & & \\
pre-admission & $85.6 \%( \pm 24.7 \%)$ & $85.7 \%( \pm 23.6 \%)$ & $<.974$ \\
preoperatively & $58.3 \%( \pm 9.7 \%)$ & $83.5 \%( \pm 11.9 \%)$ & $<0.001^{\star \star *}$ \\
intraoperatively & $76.8 \%( \pm 10.7 \%)$ & $74.4 \%( \pm 12.8 \%)$ & 0.301 \\
postoperatively & $21.2 \%( \pm 7.2 \%)$ & $47.2 \%( \pm 16.3 \%)$ & $<0.001^{\star \star *}$ \\
overall & $46.3 \%( \pm 4.9 \%)$ & $64.7 \%( \pm 9.1 \%)$ & $<0.001^{\star \star *}$ \\
\hline
\end{tabular}

SMD, Standardized Mean Difference. ${ }^{* *} \mathrm{P}<0.001$, Generalized Estimating Equation (GEE). 